\title{
NDE of CFRP composites by transient thermography
}

\author{
by W. Peng and D. Almond
}

Department of Materials Science and Engineering, University of Bath, BA2 7AY, UK e-mail: mspwp@materials.bath.ac.uk

\begin{abstract}
Investigations are presented of factors that affect the transient thermography (TT) detectabilities of defects in carbon fibre reinforced plastic (CFRP) plates. The factors studied were: heating time and its optimisation; defect size, depth and severity; image processing. A quantitative comparison was made of TT and ultrasonic C-scan measures of defect size.
\end{abstract}

\section{Introduction}

When the TT technique is used as a practical NDE method, the contrast yielded in the IR image is of major interest, where contrast is defined as the difference between the defect image temperature and temperature over a sound part of the test piece. However, this contrast depends on the temperature rise of the test piece as a whole. A more useful measure is "detectability", defined as the temperature rise on the surface above the centre of the defect $\left(\Delta \mathrm{T}_{\text {defect }}\right)$ divided by the temperature rise on the surface at a region remote from the defect region $\left(\Delta T_{\text {sound }}\right)$ or, formally:

$$
\text { Detectability }=\Delta T_{\text {defect }} / \Delta T_{\text {sound }}
$$

The advantage of using detectability is that it can be directly used as a tool to assess whether a defect can be easily detected, and it is not influenced by the total heat absorbed which is normally a difficult property to measure and control [1].

It is well known that the detectability (and the contrast) of the TT technique depend in a complex way on defect parameters and heating-dependent parameters (heating time, heating intensity, heating distribution and the time when the image is recorded). Therefore, it is necessary to study these effects quantitatively to obtain a comprehensive assessment of TT technique.

\section{Experimental system}

The $\pi$ system used for this work was an Agema Thermovision 750 infrared camera, which employed an InSb detector that was sensitive to $2-5.6 \mu \mathrm{m}$ infrared radiation. The camera's image scan rate was $6.25 \mathrm{~Hz}$ and each of the images was digitised (as $256 \times 248$ pixel arrays of 8 bit numbers) and recorded by a modular frame grabber TMS 34020 card installed in a Viglen 486-PC. Two 500 Watt flash lamps were used to heat the sample for a period of time controlled by a second PC. Mechanical shutters were positioned in front of the lamps, falling down at the end of heating to stop any "after glow" heat from the filament reaching the sample. The frame grabber was electrically triggered to record a sequence of 24 (at most) digitised thermal images after the lamps were switched off.

\section{Samples}

24-ply quasi-isotropic CFRP laminates of $3 \mathrm{~mm}$ thickness were used as the test-pieces. In these, three categories of defects were introduced: i) back-drilled cylindrical hole defects of diameters $12.7,10,8,6,4$ and $2 \mathrm{~mm}$ at depth $1.5 \mathrm{~mm}$, and $10 \mathrm{~mm}$ diameter holes at depths $0.25,0.75,1.25,1.75$ and $2.25 \mathrm{~mm}$; ii) inclusion defects of different levels of severity produced by inserting 1 to 10 layers of Teflon fabric $(10 \mathrm{~mm} \times 10 \mathrm{~mm})$ at depths $0.5,1.0$ and $1.5 \mathrm{~mm}$; iii) real impact defects induced by controlled falling weights of impact energy equivalent to $1 \mathrm{~J}$ to $6 \mathrm{~J}$. 


\section{Experimental procedures}

The samples were, one by one, mounted vertically in the focal plane of the camera. The surface of the sample facing the camera was heated for $5 \mathrm{~s}$, as this heating duration provided high detectability needed for deep defects. See Section 5.1 for details.

The detectability was quantified by extracting characteristic thermal profiles in the form of lines of digital data from image data files. To reduce the noise to an acceptable level, four sequential profiles over the centre of the defect area were averaged to provide $\Delta T_{\text {defect }}$ and $\Delta \mathrm{T}_{\text {sound. }}$.

Uneven heating was very severe in this set-up, since the samples were relatively large $(86 \mathrm{~mm} \times 150 \mathrm{~mm})$. To extract the defect area from the noisy background, for defect size measurement or calculation, special image arithmetical operations were conducted using Optimas 6.1 software package, with the help of reference images taken under the same experimental conditions for a defect-free sample. The edges of the sample were used for spatial calibration. The threshold value, which separates the defect area from the background noises, was determined on the basis of Full Width Half Maximum (FWHM) principle [2].

\section{Experimental results}

\subsection{Determination of optimal heating time}

To determine the optimal heating time, the heating time dependence of the detectabilities for back-drilled hole defects (all of $10 \mathrm{~mm}$ diameter) were measured as shown in figure 1a. The corresponding defect images under these heating times are shown in figure $1 b$. It is very clear that the detectability rises with the increasing of the heating time, and the deeper defects can only be seen when longer heating time was used. Further more, after its initial quick increase, the detectability stabilised. Further prolonging of the heating time from $3 \mathrm{~s}$ to $5.5 \mathrm{~s}$ made less improvement. Therefore, for the best defect visibility and deeper defect detection, the optimum heating time is about $4.5 \mathrm{~s}$ to $5.5 \mathrm{~s}$, which corresponds temperature rise of $2.8 \sim 3.6^{\circ} \mathrm{C}$.

\subsection{Defect type and depth effects}

Samples with back-drilled hole defects and Teflon inclusion defects at different depths were used to investigate their effects on the detectability quantitatively. The detectability evolution curves of each of these defects are shown in figure $2 a$ and $b$. They indicate that: 1) the shallower the defect, the higher the detectability; 2) the detectability reduces with the increasing of cooling time; 3 ) the Teflon inclusion defects possess lower detectability than the air hole defects and their thermal responses disappear more quickly, as a result of their lower thermal mismatch, compared with the air gap defects.

\subsection{Defect size effects}

Different sized back-drilled hole defects (all at $1.5 \mathrm{~mm}$ depth) and Teflon inclusion defects (all at $1.0 \mathrm{~mm}$ depth) were inspected. The effect of defect size on detectability is clear for the back-drilled hole defects in figure 3a. For the Teflon inclusions the effect was smaller and their detectabilities were lower, see figure $3 b$. (Only 2 and 4 ply results are shown).

\subsection{Defect severity effects}

Defects made by inserting different numbers of Teflon fabric ( $1 \sim 10$ layers, embedded together, in a rectangular step shape) were detected. Their detectability curves, see figure 4 , point out that TT technique is unable to give correct information on defect severity. 
http://dx.doi.org/10.21611/qirt.1998.012

\section{Defect extraction and size measurement}

\subsection{Image processing techniques}

As can be seen from raw IR images $\boldsymbol{a}$ and $\boldsymbol{b}$ in figure 5 , uneven heating and other background noise are significant. Therefore, defect extraction or size measurement are not possible without using special image processing techniques. The so-called $A \sim H$ image processing technique was used in this investigation. It includes subtracting a reference image (image $B$ ) from a raw defect image (image $A$ ) recorded at an earlier time to form a spatial reference image $C$. A temporal reference image (image $E$ ) is formed by subtracting a raw defect image recorded at a later time (image $D$ ) from image $A$. Image $C$ and $E$ are binarized using FWHM to set thresholds, to form image $F$ and $G$, and these images are combined using the Boolean AND operation to exclude noise. The combined result of image $F$ and $G$ is finally ANDed with the raw image $A$ to form image $H$ for defect extraction. $A \sim H$ image processing technique proved to be very helpful for defect extraction (see figure5 a" and $\boldsymbol{b}^{\prime \prime}$ ).

\subsection{Defect sizing results, compared with $\mathrm{C}$-scan technique}

The defect sizing results of real impact damages and artificial Teflon inclusions are charted in figure 6 a and $b$ respectively, and ultrasonic C-scan results are shown for comparison. The figure shows that the TT technique and ultrasonic C-scan produce images of similar size. But TT technique could not find Teflon inclusions as deep as $1.5 \mathrm{~mm}$, neither can it find the smallest $1 \mathrm{~J}$ impact defect.

\section{Conclusions}

This experimental study has confirmed the potential of TT as a practical and quantitative NDE technique. It has adequate sensitivity and similar spatial resolution to ultrasonic C-scan. But it is limited to the near surface defect. On the other hand, times of heating and image recording are of significant importance to TT NDE's detectability and image quality. Generally speaking, the earlier, unsaturated image will present a better result and provide information on a defect's relative depth.

\section{REFERENCES}

[1] CONNOLLY (M. P.) - A review of factors influencing defect detection in infrared thermography: Applications to coated material, Journal of non-destructive evaluation, Vol. 10, No. 3, 1991.

[2] ALMOND (D. P.), LAU (S.K.) - Defect sizing by transient thermography. I. An analytical treatment, J. Phys. D: Appl. Phys. 27, 1994. 
http://dx.doi.org/10.21611/qirt.1998.012

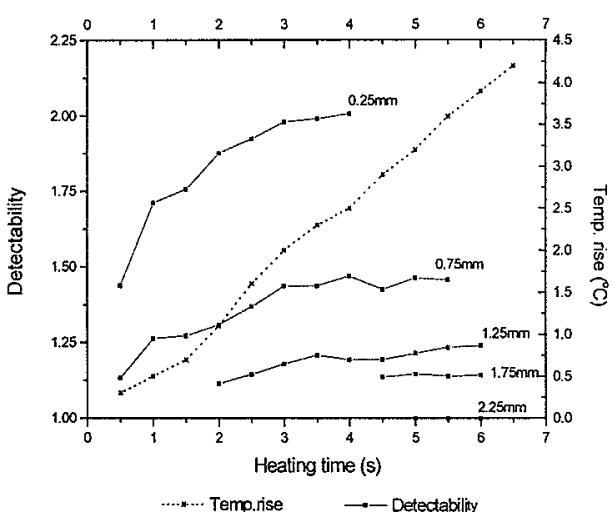

(a)
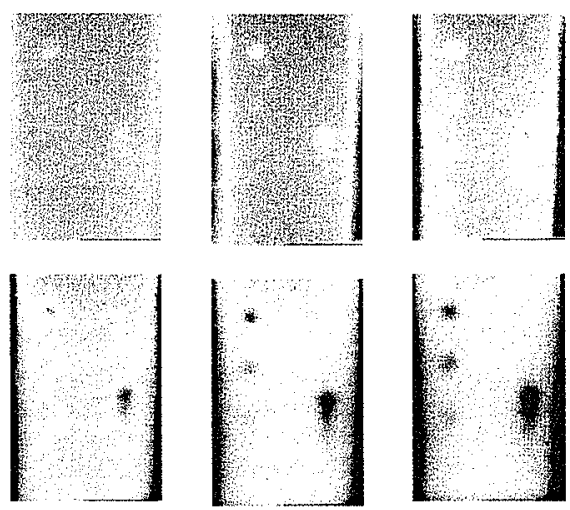

(b)

Fig. 1. (a) Temperature and detectability Vs heating time for $10 \mathrm{~mm}$ diameter backdrilled hole defects at different depths. (b) Original IR images which show that the defect detectability increases with heating time, and the deeper defect can only be found with longer heating

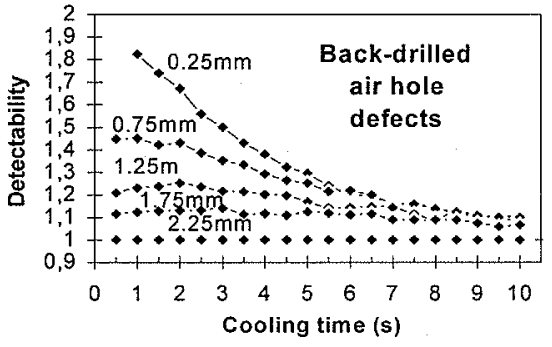

(a)

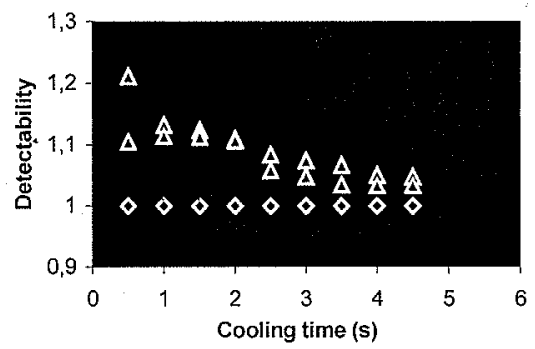

(b)

Fig. 2.(a) Detectability evolution curves of $10 \mathrm{~mm}$ diameter air hole defects at different depths. (b) Detectability evolution curves of same sized Teflon inclusion at different depths

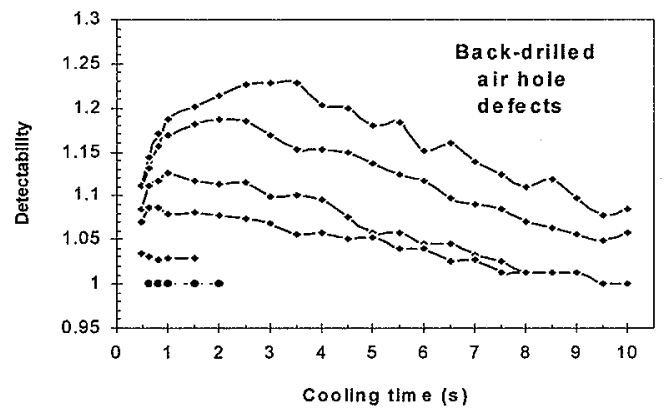

(a)

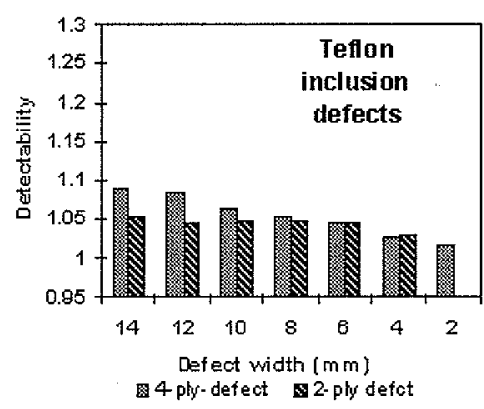

(b)

Fig. 3. Detectability evolution curves of different diameter back-drilled air hole defects (all at $1.5 \mathrm{~mm}$ depth). Detectabilities of Teflon defects of different widths

(all at $1.0 \mathrm{~mm}$ depth) 


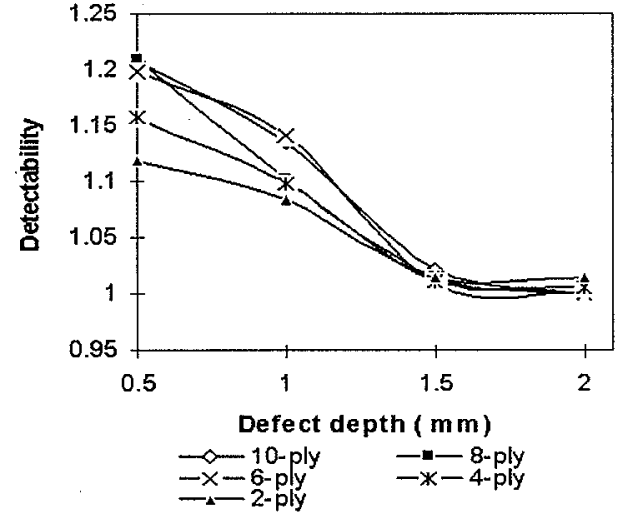

(a)

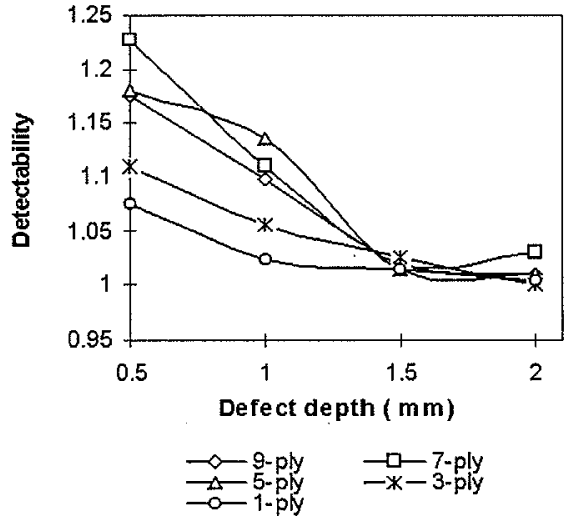

(b)

Fig. 4. (a \& b) Detectabilities of defects made by inserting together different numbers of Teflon cloth of same size at $0.5,1.0$ and $1.5 \mathrm{~mm}$ receptively. Data were extracted from the first raw. images $(0.5 \mathrm{~s})$ after 5 s heating

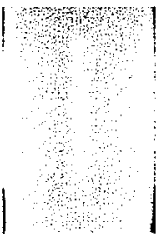

a

Original IR image of inclusion defect

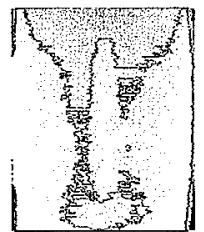

$a^{3}$

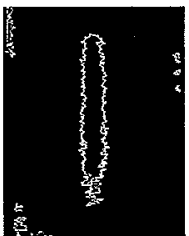

a"

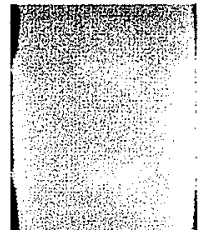

b

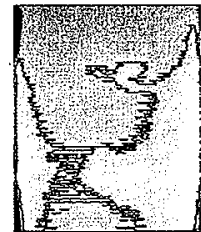

b'

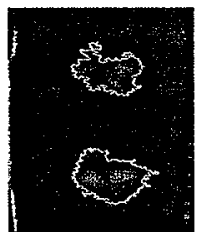

b"

Defect Extraction Original IR extraction by after image image of FWHM based on a processing (A H) impact damage
Defect extraction by FWHM based on $b$
Extraction

after image processing (A H)

Fig. 5. Original defect images and defect extraction

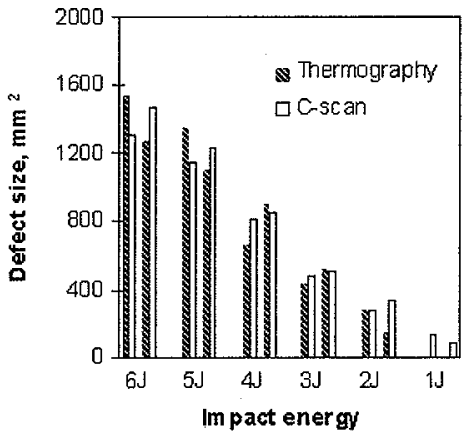

(a) Impact defects

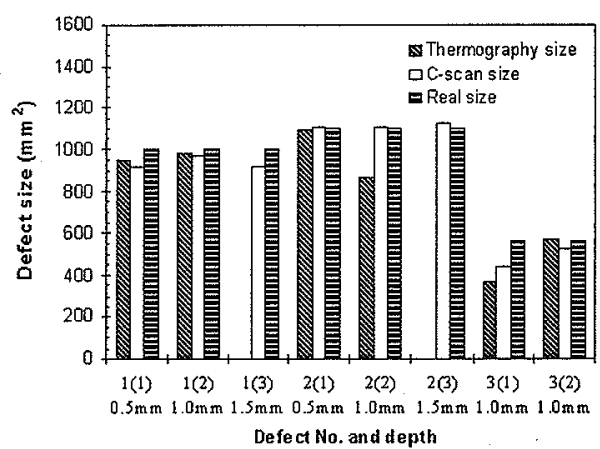

(b) Teflon inclusion defects

Fig. 6. Defect sizing results compared with ultrasonic C-scan measurement 